\title{
in a cohort of patients with diabetes mellitus type 1 and comorbid chronic kidney disease in Belarus
}

\author{
A. Sazonava, T. Mokhort, M. Astapovich, ${ }^{*}$ S. Petrenko**
}

Belarusian State Medical University, Minsk City Endocrinological Dispensary, ${ }^{*}$ International Sakharov Environmental University**

OBJECTIVES

According to World Health
Organization (WHO) iodine deficiency
(ID) occurs due to lack of dietary
iodine and results in impaired
biosynthesis of thyroid hormones
and/or thyroid enlargement.
The aim was to assess iodine
supplementation and thyroid structure
changes in a cohort of patients with
diabetes type 1 (DM 1) at different
stages of comorbid chronic kidney
disease (CKD) after achievement of
adequate iodine status in Belarus.

\section{METHODS}

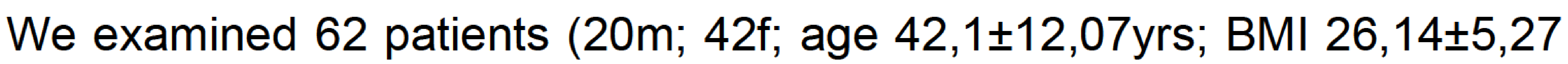

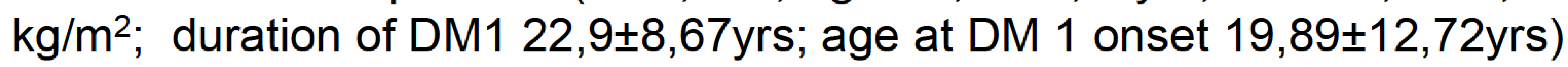
at $\mathrm{CKD}$ stages $1,2,3,5(\mathrm{~N}=28 ; 21 ; 12 ; 1$, respectively $)$. GFR was estimated using Cockcroft-Gault formula.

Urinary iodine excretion in urine morning sample was measured with the use of cerium-arsenite method approved $\mathrm{WHO}$ as a standard.

ID is defined as a median urinary iodine concentration less than $\mathbf{5 0} \boldsymbol{\mu} / \mathrm{l}$ in a population.

All data concerning ultrasound structure of thyroid gland were determined as normal, reduced, increased volume ( $\mathrm{N}$ Vol, redVol, incrVol), homogeneity (hypo-, hyper-, homogeneous), local pathology (abs, single, multiple).

Nonparametrics and descriptive statistical methods were used.
\end{abstract}

\section{RESULTS}

Thyroid gland volume



CONCLUSIONS

ID was not registered in examined population of patients that demonstrates the effectiveness of iodine prophylaxis. Revealed a direct correlation between the level of urinary iodine excretion and CKD stage can lead to a variety of structural changes in the thyroid gland which requires close monitoring of patients with reduced renal function.
In the examined group evaluating iodine supplementation in morning urine samples a lack of ID was registered - a median urinary iodine excretion at the time of survey was $115,5[58,0 ; 241,2]$ $\mathbf{m k g} / \mathrm{l}$. Comparative analysis of patients in the subgroups according to CKD stages revealed reliable differences in level of urinary iodine excretion $(p=0,0133: p<0,05)$, total volume of thyroid gland $(p=0,0325)$, and in homogeneity of thyroid gland $(p=0,040)$. Urinary iodine excretion in morning sample correlates with urea plasma level $(r=-0,309: p<0,05)$, GFR $(r=0,420)$, CKD stage $(r=-0,323)$. Total volume of thyroid gland correlates with plasma urea $(r=0,313)$ and creatinine levels $(r=0,259)$. Strong correlation was revealed between homogeneity and age at DM 1 onset $(r=0,292)$. Structural changes in thyroid gland such as hypoechoic structure were reported in 24 patients $(38,71 \%)$.

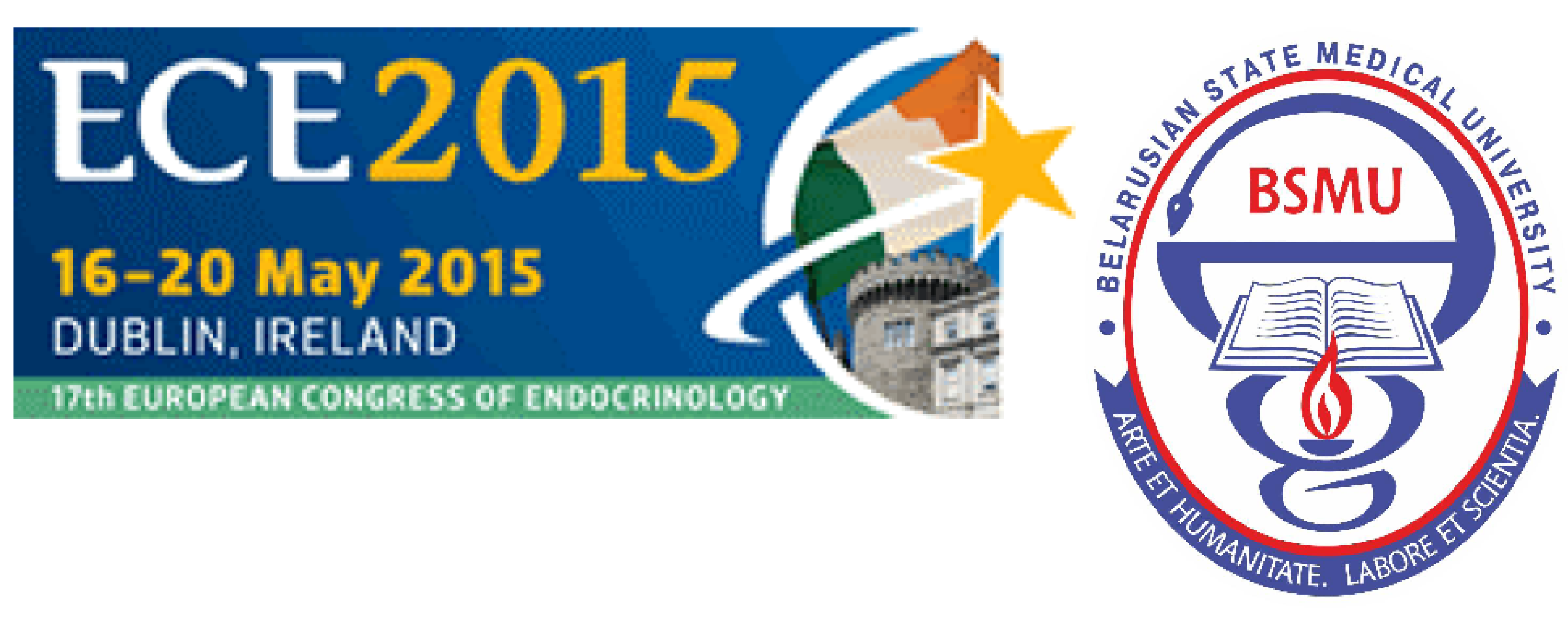

